\title{
CaracterizaÇÃO dos SINTOMAS DO Transtorno de Estresse Pós-Traumático (TEPT) EM MENINAS VÍTIMAS DE ABUSO SEXUAL
}

\author{
Luisa Fernanda Habigzang* \\ Jeane Lessinger Borges** \\ Débora Dalbosco Dell'Aglio*** \\ Silvia Helena Koller ${ }^{* * * *}$
}

\section{Resumo}

O presente artigo descreve a presença de sintomas ou do diagnóstico do Transtorno do Estresse Pós-Traumático (TEPT) em meninas vítimas de abuso sexual. Além disso, buscou-se comparar o uso de dois instrumentos de avaliação diagnóstica do TEPT infantil em duas amostras clínicas. No Estudo I ( $\mathrm{n}=40$ meninas), os sintomas foram avaliados através de uma entrevista clínica, baseada no DSM-IV/SCID/TEPT. E no Estudo II ( $\mathrm{n}=15$ meninas), através da versão brasileira da K-SADS-PL/TEPT. Os resultados apontaram semelhanças na presença do diagnóstico de TEPT nas duas amostras, próxima a 70\%, apesar de os instrumentos indicarem diferenças específicas quanto à manifestação dos critérios $\mathrm{C}$ (evitação e entorpecimento) e critério D (excitabilidade aumentada). Pequenas diferenças na estrutura e na forma de aplicação dos instrumentos foram observadas. Conclui-se que ambos se aplicam à pesquisa e à avaliação clínica do TEPT infantil.

Palavras-chave: abuso sexual; estresse pós-traumático; entrevista clínica.

* Psicóloga, Mestre e doutoranda em Psicologia (UFRGS).

** Psicóloga, Mestre em Psicologia (UFRGS).

*** Doutora em Psicologia do Desenvolvimento (UFRGS), docente do Programa de PósGraduação em Psicologia (UFRGS).

**** Doutora em Educação (PUCRS) e docente do Programa de Pós-Graduação em Psicologia (UFRGS). 


\begin{abstract}
Characterization of symptoms of Posttraumatic Stress Disorder (PTSD) IN GIRLS VICTIMS OF SEXUAL ABUSE

The present paper describes the presence of symptoms or diagnosis of Posttraumatic Stress Disorder (PTSD) in girls victims of sexual abuse, through two clinical interviews. Moreover, the use of two instruments of assessment of child PTSD diagnosis in two clinical samples has been compared. In Study I ( $n=40$ girls) symptoms were assessed through clinical interview, based on DSM-IVISCID/TEPT, and in Study II ( $n=15$ girls) through the Brazilian version of K-SADS-PL/TEPT. The results pointed out similarities concerning the presence of PTSD diagnosis in both samples, around 70\%, although instruments showed specific differences concerning the manifestation of criteria $C$ (avoidance and numbing) and criteria $D$ (excessive arousal). Small differences in the instruments' structure and application were observed. It has been concluded that both instruments can be applicable to research and to clinical assessment of child PTSD.
\end{abstract}

Keywords: sexual abuse; posttraumatic stress; clinical interview.

\title{
INTRODUÇÃO
}

O presente artigo tem como objetivo caracterizar a presença de sintomas ou do diagnóstico do Transtorno de Estresse Pós-Traumático (TEPT), em meninas vítimas de abuso sexual. Além disso, busca comparar o uso de duas entrevistas clínicas utilizadas na avaliação do TEPT. Inicialmente, aspectos conceituais sobre o TEPT são apresentados. Em seguida, os resultados de dois estudos empíricos são discutidos, apresentando características do quadro de estresse pós-traumático identificado nas participantes.

O abuso sexual infantil (ASI) tem sido considerado um problema de saúde pública devido a sua alta prevalência e ao impacto negativo de suas consequências no desenvolvimento físico, emocional, cognitivo e social das vítimas (Briere \& Elliott, 2003; Gomes, Deslandes, Veiga, Bhering \& Santos, 2002; Tyler, 2002). Algumas crianças e adolescentes vítimas de ASI podem apresentar mínimas alteraçōes comportamentais e emocionais, enquanto outras desenvolvem transtornos psicológicos significativos (Elliott \& Carnes, 2001; Runyon \& Kenny, 2002; Saywitz, Mannarino, Berliner \& Cohen, 2000). Além disso, crianças e adolescentes podem desenvolver psicopatologias, tais como: transtornos de humor, de ansiedade e hiperatividade e déficit de atenção (Briere \& Elliott, 2003; Duarte \& Arboleda, 2004; Paolucci, Genuis \& Violato, 2001).

O transtorno de estresse pós-traumático - TEPT é a psicopatologia mais citada como decorrente do abuso sexual (Ackerman, Newton, McPherson, Jones 
\& Dykman, 1998; Cohen, 2003; Famularo, Fenton, Kinscherff \& Augustyn, 1996; Ruggiero, McLeer \& Dixon, 2000). A prevalência deste transtorno pode variar entre 20 a $70 \%$ dos casos de crianças vítimas de ASI (Nurcombe, 2000). Nos Estados Unidos, a presença do diagnóstico de TEPT foi de 36,3\% entre as crianças abusadas sexualmente (Ruggiero et al., 2000). Em outro estudo, no Canadá, foi de $46 \%$ numa amostra com meninas vítimas de ASI (Collin-Vézina \& Hébert, 2005). De um modo geral, observa-se uma elevada prevalência de TEPT em casos de ASI, se comparada à estimativa de que na população geral varie de $6 \%$ nos homens a $14 \%$ nas mulheres (Breslau, 2002).

O TEPT é um transtorno de ansiedade que ocorre após a exposição a um evento traumático. Nele a avaliação subjetiva da vítima, envolvendo resposta de pavor e medo (Critério A), está interligada a sintomas de revivência, evitação e excitabilidade fisiológica aumentada; ao tempo (superior a um mês, Critério E); e ao prejuízo funcional (Critério F), segundo o Manual Diagnóstico e Estatístico dos Transtornos Mentais - DSM-IV-TR (American Psychiatric Association, 2002).

Três categorias de sintomas são descritas, a saber: 1) re-experiência intrusiva do trauma (um ou mais sintomas, Critério B); 2) esquiva persistente de estímulos associados com o trauma e entorpecimento da reatividade geral (três ou mais sintomas, Critério C); 3) sintomas persistentes de excitabilidade fisiológica (dois ou mais sintomas do Critério D (American Psychiatric Association, 2002). O critério de re-experiência traumática envolve lembranças intrusivas e recorrentes que podem ocorrer sob a forma de sonhos aflitivos e pesadelos. São carregadas de forte componente afetivo, sendo, portanto, associadas à angústia e ao sofrimento intenso do paciente (Câmara Filho \& Sougey, 2001). A manifestação de flashbacks é caracterizada pela sensação de a vítima sentir-se como se estivesse revivendo o evento traumático no momento atual de sua vida.

O comportamento de esquiva, em geral, interfere nas atividades cotidianas da pessoa devido à alta energia emocional empenhada na aquisição de lembranças e sentimentos relacionados ao trauma. Percebe-se, ainda, o entorpecimento emocional, o qual pode ser caracterizado pela dificuldade do(a) paciente com TEPT descrever, expressar e ganhar afeto. Sintomas de taquicardia, respiração ofegante, formigamentos, sudorese, tonturas, dores abdominais, entre outros, acompanham as lembranças traumáticas e a evitação cognitiva e emocional do trauma. A hipervigilância, que é caracterizada como "estar em guarda e atento" aos estímulos externos, coloca a vítima num estado persistente de ameaça, em que o ambiente sempre é considerado como um lugar inseguro e imprevisível. Por último, resposta de sobressalto exagerada também é comum nas pessoas com 
TEPT, isto é, as vítimas facilmente se assustam com qualquer estímulo (Câmara Filho \& Sourgey, 2001).

Embora critérios para adultos sejam também utilizados para o diagnóstico infantil, algumas modificações específicas à sintomatologia do TEPT em crianças devem ser observadas (Dyregrov \& Yule, 2006; Pynoos, 1992). Crianças traumatizadas desenvolvem sintomas de hiperatividade, dificuldade de atenção, ansiedade de separação, medos, queixas psicossomáticas e, frequentemente, retrocesso no desenvolvimento (Kaminer, Seedat \& Stein, 2005). Reencenação do trauma, através de brincadeiras e jogos repetitivos, sonhos traumáticos recorrentes ou pesadelos sem conteúdo identificável, e interesse diminuído em atividades habituais podem ser caracterizados como sintomas de TEPT na infância (Pynoos, 1992).

Os sintomas clínicos do TEPT infantil podem variar de acordo com a etapa do desenvolvimento (Dyregrov \& Yule, 2006; Kaminer et al., 2005). Por exemplo, crianças pequenas tendem a apresentar mais sintomas de agressividade e hiperatividade. Já adolescentes tendem a apresentar sintomas mais próximos daqueles dos adultos. Além disso, atualmente tem-se discutido a necessidade de uma revisão dos critérios (sintomas e número de itens por critérios) para o diagnóstico de TEPT infantil. Estudo recente sugeriu redução de três para um sintoma de evitação do critério C, para crianças em idade pré-escolar (Scheeringa, Wright, Hunt \& Zeanah, 2006).

Dessa forma, a avaliação do TEPT infantil torna-se uma tarefa minuciosa para os clínicos. Alguns instrumentos têm sido utilizados nesta tarefa, entre eles entrevistas clínicas estruturadas e escalas. Hawkins e Radcliffe (2006) realizaram uma revisão de literatura referente aos principais instrumentos utilizados na avaliação do diagnóstico de TEPT infantil, englobando 65 estudos publicados entre 1995 e 2004 . Seis instrumentos foram citados por estes autores, incluindo: o Diagnostic Interview for Children and Adolescents (DICA); Kiddie Schedule for Affective Disorders and Schizophrenia for School-Age Children-Present and Lifetime Version (K-SADS-PL); Clinician-Administered PTSD Scale for Children and Adolescents (CAPS-CA); Impact of Events Scale-Revised (IES-R); Child PostTraumatic Stress Disorder Reaction Index (CTPSD-RI); PTSD Symptom Scale (PPS) e o Trauma Symptom Cheklist for Children (TSCC). Ainda pode-se citar o Child PTSD Cheklist, o Child and Adolescent Trauma Survey (Kaminer, Seedat \& Stein, 2005) e o Childhood PTSD Interview-Child Form como instrumentos de avaliação para sintomas de TEPT infantil (Perrin, Smith \& Yule, 2000).

No Brasil, há uma escassez de instrumentos disponíveis à avaliação do TEPT, tanto para a população adulta quanto infantil. A versão brasileira da K-SADS-PL (Brasil, 2003; Kaufman et al., 1997) inclui um módulo de perguntas referentes a 
avaliação clínica do TEPT. Além disso, Del Ben et al. (2001) traduziram e adaptaram a Entrevista Clínica Estruturada para o DSM-IV (SCID-TEPT).

A partir destas considerações iniciais, o presente estudo tem como objetivo: (a) caracterizar a presença de sintomas e/ou do diagnóstico do TEPT em meninas vítimas de ASI; e (b) comparar o uso de duas entrevistas clínicas estruturadas utilizadas na avaliação do TEPT infantil disponíveis no Brasil.

\section{Método}

Os dois estudos (descritos a seguir) consideram o abuso de forma ampla, incluindo contatos sexuais com e sem penetração. A suspeita e/ou a ocorrência de abuso sexual foi avaliada pela equipe técnica dos serviços em que foram realizadas as entrevistas. A avaliação do TEPT foi realizada através de duas entrevistas clínicas diferentes, nas quais o conteúdo das questões é baseado nos critérios definidos pelo DSM-IV-TR (American Psychiatric Association, 2002), ou seja, um ou mais sintomas presentes para o Critério $\mathrm{B}$; três ou mais sintomas presentes para o Critério C; e dois ou mais sintomas do Critério D. Ao final da pesquisa, todas as participantes receberam atendimento psicológico no próprio local da entrevista ou na rede de saúde mental do município.

\section{ESTUDO I}

\section{Participantes}

Participaram deste estudo 40 meninas com idade entre nove e 16 anos $(M=1,28, D P=1,89)$, residentes de uma cidade da região metropolitana de Porto Alegre/RS. Os critérios de inclusão no estudo foram: ter sido vítima de ASI (ao menos um episódio); a família consentir com a participação da menina na pesquisa. As meninas se encontravam em processo de avaliação psicológica num serviço público especializado em casos de ASI. A coleta de dados ocorreu, em média, após um mês da revelação do abuso. Nenhuma das participantes fazia uso de medicação psicotrópica.

O contexto do abuso sexual foi intrafamiliar em 30 casos $(75 \%)$, extrafamiliar em seis casos (15\%) e intra e extrafamiliar em quatro casos (10\%). Em 82,5\% dos casos houve episódios múltiplos de abuso, e em 17,5\% a violência se configurou como episódio único. A forma de abuso sexual envolveu contato físico sem estupro 
em $65 \%$ dos casos, estupro em $30 \%$ e assédio verbal ou exibicionismo, sem contato físico, em 5\%. A idade de início do abuso sexual ocorreu em média aos 8,97 anos $(D P=2,12)$. A duração da violência foi de mais de um ano em $37,5 \%$ dos casos e contatou-se a presença de outras formas de violência (física e psicológica) em $92,5 \%$ da amostra.

\section{INSTRUMENTOS E PROCEDIMENTOS DE COLETA DE DADOS}

1) Entrevista semiestruturada inicial constituída por duas partes: na primeira parte, o objetivo foi estabelecer um vínculo terapêutico com a participante, criando um espaço seguro baseado em uma relação de confiança. A segunda parte está baseada na entrevista publicada pelo "The Metropolitan Toronto Special Committee on Child Abuse" (1995), traduzida para o português e adaptada por Kristensen (1996). Esta entrevista tem como principal objetivo obter o relato da participante com relação ao abuso sexual, bem como mapear a frequência e a dinâmica dos episódios abusivos.

2) Entrevista estruturada com base no DSM IV/SCID para avaliação do TEPT: Os critérios diagnósticos, estabelecidos pelo Manual Diagnóstico e Estatístico dos Transtornos Mentais (DSM), são utilizados como base para identificação da presença dos sintomas que compõem o transtorno (re-experienciação do evento traumático; esquiva de estímulos associados com o trauma; e, sintomas de excitabilidade aumentada). A tradução para o português desta entrevista foi desenvolvida por Del Ben et al. (2001). Alteraçôes foram feitas na linguagem utilizada na entrevista para facilitar a compreensão das perguntas pelas crianças e adolescentes, tendo como base os resultados do estudo piloto, no qual a entrevista foi aplicada em 10 meninas com idade entre nove e 12 anos (Habigzang, 2006).

O projeto foi submetido e aprovado pelo Comitê de Ética da Universidade Federal do Rio Grande do Sul. As participantes foram encaminhadas pelo Conselho Tutelar, abrigos, Juizado da Infância e Juventude e Programa Sentinela do município no qual a pesquisa foi realizada. As participantes foram incluídas mediante a assinatura do Termo de Consentimento Livre e Esclarecido de seus responsáveis e pelo seu consentimento. As entrevistas foram realizadas com as meninas em dois ou três encontros com frequência semanal e duração de 1 hora. A entrevista inicial foi gravada e transcrita. A entrevista que avaliou TEPT foi pontuada pela entrevistadora e depois reavaliada por outra pesquisadora da equipe. 
ESTUDO II

\section{PARTICIPANTES}

Participaram deste estudo 15 meninas vítimas de abuso sexual intra e/ou extrafamiliar, com idades de sete anos a 13 anos $(M=9,50 ; D P=1,58)$, residentes de Porto Alegre/RS (62,5\%) ou municípios da regiāo metropolitana (37,5\%). Foram utilizados como critérios de inclusão: ser vítima de abuso sexual intra ou extrafamiliar; o abuso ter ocorrido há mais de um mês; estar no máximo na segunda sessão de avaliação psicológica e/ou atendimento psicoterápico; e contar com a presença de um familiar não-abusador ou cuidador legal, responsável pela participante da pesquisa. As participantes se encontravam em atendimento num serviço de atendimento psicológico de um hospital público. A coleta de dados foi realizada num período entre 1 e 36 meses após o último episódio de ASI $(M=11,20 ; D P=10,46)$. Nenhuma participante estava fazendo uso de medicação psicotrópica.

A maioria dos casos envolveu situações de ASI intrafamiliar (12 intrafamiliar e três casos extrafamiliar), sendo que a idade média de início à exposição abusiva foi de 7 anos e 6 meses $(D P=2,50)$. Os episódios únicos incluíram 26,7\% dos casos, enquanto os episódios múltiplos $73,3 \%$. A duração do ASI variou entre episódio único e dois anos $(M=10,53$ meses; $D P=11,09)$. Em relação ao tipo de abuso sexual sofrido, em $53,3 \%$ envolveram toques e carícias e em $46,7 \%$ abuso sexual com penetração. Entre os principais abusadores estão: padrasto $(26,7 \%)$, tio $(26,7 \%)$ e avô $(13,3 \%)$. De um modo geral, foi observada a presença de segredo ao longo do período em que a menina estava sendo vítima de ASI, sendo que 46,7\% sofreram algum tipo de ameaça por parte do abusador e $26,7 \%$ ganharam algum tipo de barganha para manter o segredo (balas e presentes).

\section{INSTRUMENTOS E PROCEDIMENTOS DE COLETA DE DADOS}

1) Questionário sobre Saúde e Dados Demográficos: avaliou indicadores sociodemográficos das participantes (idade, escolaridade, cidade de origem) e questões referentes à saúde mental (transtornos psiquiátricos, neurológicos e uso de medicações). Características do ASI (idade do primeiro episódio, tempo de exposição, presença de ameaça, proximidade afetiva com o abusador e encaminhamentos legais sobre o caso) e do contexto da revelação (para quem a criança revelou pela primeira vez o abuso, reação da família e presença de suporte familiar após a revelação) foram avaliadas. Este questionário foi realizado junto às mães 
e/ou cuidadores legais responsáveis pelas meninas, em uma sessão individual, após rapport inicial com a pesquisadora.

2) Versão brasileira da Schedule for Affective Disorders and Schizophrenia for School Aged-Children, Kiddie - Sads (K-SADS-PL) (Brasil, 2003; Kaufman et al., 1997): consiste em uma entrevista clínica diagnóstica, semiestruturada, baseada nos critérios do DSM-III e do DSM-IV, para verificar e registrar episódios psicopatológicos, passados ou correntes em crianças e adolescentes (6 a 18 anos). A K-SADS-PL é administrada entrevistando-se um dos pais/cuidadores e a criança ou adolescente. Ao final do processo de avaliação, obtém-se uma pontuação conclusiva (resumo total). Para este estudo, foi utilizado o módulo referente à entrevista de rastreamento diagnóstico do TEPT. Esta entrevista clínica foi realizada tanto com as mães e/ou cuidadores quanto com as meninas, de forma individual. Para a avaliação do diagnóstico de TEPT foi considerada a pontuação total dos itens dos critérios do TEPT para o momento atual. Foi utilizada, ainda, a lista de eventos traumáticos, propostos pela K-SADS-PL, como medida de avaliação de outras situações estressoras que pudessem contribuir na etiologia do TEPT.

Este estudo foi submetido e aprovado junto aos Comitês de Ética do Hospital Materno Infantil Presidente Vargas (HMIPV) e da Universidade Federal do Rio Grande do Sul (UFRGS). Foi solicitada a assinatura do Termo de Consentimento Livre e Esclarecido, obtido junto às mães e/ou responsáveis legais pelas meninas. Foram realizadas duas sessões junto à mãe e/ou responsável legal pela criança. Posteriormente, uma terceira sessão foi feita com as meninas, sendo que estas responderam apenas os critérios de TEPT, propostos pela K-SADS-PL, em uma entrevista individual.

\section{Resultados}

Os resultados são apresentados a seguir conforme as variáveis de interesse. No primeiro momento, os dados referentes à sintomatologia do TEPT em meninas vítimas de abuso sexual foi caracterizada e, no segundo, foram comparadas as características dos dois instrumentos clínicos para a avaliação do TEPT.

\section{DESCRIÇÃO DA SINTOMATOLOGIA DO TEPT EM VITIMAS DE ASI}

A Tabela 1 (ver anexo) apresenta a prevalência geral do diagnóstico e a média de sintomas por critérios do TEPT nas duas amostras. Observa-se que 
a prevalência de TEPT foi elevada nos dois estudos. No entanto, foi observada diferença nas médias de sintomas entre os critérios do TEPT, sendo que a média dos critérios B e C foi superior no Estudo I e a média do critério D foi superior no Estudo II.

Foi analisada a sintomatologia do TEPT nas participantes com diagnóstico completo de TEPT (de acordo com os critérios do DSM-IV-TR) e também nas que não apresentaram os critérios mínimos para o diagnóstico (Tabela 2; ver anexo). Entre as participantes com diagnóstico, observou-se uma média de 11,38 sintomas no Estudo I e de 10,73 sintomas no Estudo II. Verificou-se, ainda, um maior número de sintomas nos critério $\mathrm{B}$ e $\mathrm{C}$ no Estudo I e maior número de sintomas no critério D do Estudo II. Entre as meninas que não apresentaram o diagnóstico completo, observou-se uma média de 6,27 sintomas no Estudo I e de 5,25 sintomas no Estudo II, e uma média superior nos critérios C e D no Estudo I, enquanto a média no critério B foi superior no Estudo II. Além disso, no Estudo I a média do critério D foi superior se comparada ao Estudo II.

Os itens dos instrumentos utilizados nos Estudos I e II estão apresentados na Tabela 4 (ver anexo). Uma versão adaptada da entrevista DSM IV/SCID para avaliação do TEPT foi utilizada no Estudo I, sendo esta composta por um total de 16 itens. A versão brasileira da K-SADS-PL, contemplando o módulo do TEPT, foi utilizada no Estudo II, constituída por 20 itens.

Uma análise comparativa dos dois instrumentos utilizados nos Estudos I e II apontou algumas diferenças quanto à apresentação escrita dos itens referentes aos critérios B e C do TEPT. No critério B, referente aos sintomas de revivência do evento traumático, apesar da diferença no número de questões, todos os sintomas referidos pelo DSM-IV-TR para o critério de revivência são investigados pelos dois instrumentos.

Verificou-se, no entanto, que a questão 3, correspondente a agir ou sentir como se o evento traumático estivesse ocorrendo novamente, é realizada de forma diferente. No instrumento do Estudo I, a questão investiga a sensação de que o evento esteja acontecendo quando não está acontecendo. Já no instrumento do Estudo II, o foco da pergunta está na reencenação específica do trauma, frequente em crianças pequenas. Além disso, verificou-se um número diferente de questóes sobre o critério B nos instrumentos dos Estudos I e II. A pergunta 4 do instrumento I equivale às perguntas 5 e 6 do instrumento II. E a pergunta 5 do instrumento I equivale à pergunta 4 do instrumento II. No critério $\mathrm{C}$, referente aos sintomas de esquiva e entorpecimento, verificou-se que, embora o número de questôes seja o mesmo nos dois instrumentos, o conteúdo das perguntas difere para avaliação dos sintomas. As perguntas 3, 4, 5, 6 e 7 apresentam maiores diferenças, sendo 
que estas questōes referem-se principalmente aos sintomas de entorpecimento (ver Tabela 3 no anexo).

No critério D, que avalia sintomas de excitabilidade aumentada, conforme apresentado na Tabela 4 (ver anexo), observou-se maior semelhança entre os instrumentos, tanto em relação ao número de perguntas quanto em relação ao conteúdo destas. A variação no conteúdo das perguntas não modifica, no entanto, o sentido do sintoma que está sendo investigado.

\section{Discussão}

O presente estudo avaliou a presença do diagnóstico de TEPT em meninas vítimas de abuso sexual. Além disso, analisou duas entrevistas clínicas utilizadas na avaliação do TEPT infantil. Alta prevalência do diagnóstico de TEPT foi encontrada neste estudo, corroborando estudos anteriores que indicam uma alta manifestação deste transtorno em crianças vítimas de ASI (Collin-Vézina \& Hébert, 2005; Ruggiero et al., 2000).

A manifestação do TEPT encontrada neste estudo se aproximou da estimativa proposta por Nurcombe (2000), sugerindo que a prevalência de TEPT em vítimas de ASI pode variar entre $20 \%$ a $70 \%$ dos casos. Ressalta-se, contudo, que a literatura tem descrito a presença de diferentes sintomas clínicos em crianças vítimas de ASI. Isto é, há uma heterogeneidade de consequências negativas ao desenvolvimento emocional em crianças vítimas de ASI (Kendall-Tackett, Williams, \& Finkellor, 1993; Tyler, 2002).

Desta forma, a presença dos sintomas e/ou do diagnóstico do TEPT deve ser melhor compreendida, dentro da proposta do "Modelo Multifacetado do Trauma" (Kendall-Tackett et al., 1993), no qual o TEPT é considerado uma das sequelas do ASI, mas não se limitando a ele. Busca-se ainda limitar qualquer visão determinista do tipo causa-efeito, uma vez que a exposição a um evento traumático, como pode ser considerado o ASI, não é suficiente para causar o TEPT (Perrin et al., 2000).

Ao considerar a pontuação total de sintomas, os resultados indicam que o diagnóstico de TEPT não seria alterado se os avaliadores usassem um ou outro instrumento clínico, considerando a pontuação da amostra total e da amostra com TEPT completo. No entanto, houve diferenças na distribuição das médias por critérios do TEPT. Observou-se que em relação aos critérios B e C, a média foi superior no Estudo I se comparada ao Estudo II. Em relação ao critério D, esta foi superior no Estudo II. 
Sugere-se que por um lado a versão adaptada da SCID/TEPT pareceu ter uma melhor sensibilidade para avaliação dos sintomas do Critério $\mathrm{C}$ (esquiva e entorpecimento). Por outro, a K-SADS-PL/TEPT foi mais sensível para discriminar os sintomas do Critério D (excitabilidade fisiológica aumentada) entre as participantes deste estudo com TEPT positivo. A linguagem utilizada na formulação das questões dos instrumentos pode ter facilitado a compreensão das participantes, contribuindo para uma média mais elevada em determinado critério de sintomas.

Além disso, os dados indicam que as duas entrevistas clínicas conseguem diferenciar as participantes com e sem TEPT, uma vez que a pontuação total das meninas com TEPT completo é o dobro daquelas sem o quadro, revelando uma elevada sensibilidade e especificidade. Nesse sentido, em torno de $27 \%$ das participantes entrevistadas (na amostra total) não apresentaram o diagnóstico de TEPT. Neste subgrupo, contudo, houve uma maior presença dos sintomas do Critério B (Estudo II) e do Critério D (Estudo I) do TEPT. Em relação ao número total de sintomas, este se manteve em torno de cinco sintomas dos 17 exigidos pelo DSM-IV.

Em relação às participantes sem o diagnóstico de TEPT, é importante ressaltar que nem todas as crianças vítimas de ASI desenvolvem sintomas ou psicopatologias. As reações emocionais, associadas à exposição a situações traumáticas, englobam uma heterogeneidade de alterações emocionais e comportamentais (Kendall-Tackett et al., 1993; Tyler, 2002), bem como a presença de outros quadros psicopatológicos, incluindo o Transtorno de Estresse Agudo (TEA) e o Transtorno do Estresse Extremo Não Especificado. A presença de fatores de proteção pode favorecer uma adaptação positiva para algumas crianças vítimas de ASI, apesar de estarem expostas a um contexto de significativa adversidade.

Diferença na faixa etária das participantes dos dois estudos pode ter contribuído para a diferença de sintomas encontrados nos critérios $\mathrm{C}$ e $\mathrm{D}$ entre as duas amostras. A idade entre as participantes do Estudo I variou entre 9 e 16 anos, enquanto que as do Estudo II variou entre 7 e 13 anos. Assim, os resultados podem estar enviesados pela capacidade cognitiva da criança em compreender o seu estado interno (imaturidade emocional), principalmente no que refere aos sintomas de revivência e evitação (Dyregrov \& Yule, 2006; Kaminer et al., 2005). Além disso, a presença de sintomas de TEPT pode variar conforme a etapa do desenvolvimento. Nesse sentido, crianças podem apresentar maiores sintomas de reencenação do trauma (seja na brincadeira, seja no desenho), dificuldade de concentração e de memória, enquanto que adolescentes podem apresentar uma sintomatologia próxima à dos adultos e sentimentos de futuro abreviado (Dyregrov \& Yule, 2006; Perrin et al., 2000; Scheeringa et al., 2006). 
Os instrumentos utilizados no presente estudo não abarcam em seus critérios a ampla variedade de sintomas de TEPT infantil citados pela literatura. Embora a $\mathrm{K}-\mathrm{SADS}$-PL/TEPT inclua questōes referentes a brincadeiras repetitivas e reencenação do trauma, que são uma característica específica do Critério B em crianças, os demais itens mantêm uma maior proximidade com os critérios de TEPT para adultos. Desta forma, observa-se ausência de itens voltados à manifestação de sintomas de agressividade, hiperatividade, ansiedade de separação e novos medos, os quais são referidos na literatura como tipos esperados de reações emocionais e comportamentais de crianças que vivenciaram experiências traumáticas.

Assim, apesar de os instrumentos mostrarem-se adequados para a identificação do TEPT, poderiam ainda incluir outras reaçōes típicas de crianças, relatadas na literatura, assim como procurar abranger a heterogeneidade dos sintomas através de itens mais específicos para esta faixa etária.

Outra limitação dos instrumentos utilizados neste estudo refere-se à avaliação da presença de apenas um evento traumático para o desenvolvimento dos sintomas do TEPT. A maioria das crianças vítimas de ASI é exposta a múltiplos eventos estressores e diferentes categorias de maus-tratos. Realizar a avaliação do TEPT baseada em apenas um evento traumático limita ou enviesa a avaliação, pois não permite verificar diferenças entre trauma crônico e agudo ou múltiplos traumas e o diagnóstico do TEPT (Hawkins \& Radcliffe, 2006).

Em relação ao uso da entrevista clínica K-SADS-PL/TEPT, as questões são dirigidas tanto ao momento atual quanto ao passado, e são entrevistados tanto a criança quanto os pais e/ou cuidadores. A participação dos pais na avaliação dos sintomas de TEPT pode influenciar no resultado final da entrevista. Pine e Cohen (2002) ressaltam que uma maior valorização dos sintomas e consequências pode ser observada pelos pais, devido a seu próprio sofrimento psíquico diante da revelação do abuso sexual sofrido pelas suas filhas. Logo, as respostas dadas por estes podem estar enviesadas. Por outro lado, estas informações dos pais podem contribuir na identificação de sintomas sobre os quais as crianças não apresentam consciência.

\section{CONSIDERAÇÕES FINAIS}

Os resultados encontrados nos dois estudos descritos confirmam pesquisas que indicam o TEPT como um quadro psicopatológico com elevada manifestação em crianças e adolescentes. Em relação ao uso dos instrumentos, observou-se que 
a aplicação da versão adaptada da SCID/TEPT e da entrevista clínica K-SADSPL/TEPT indicou um resultado semelhante nas duas amostras. Pode-se observar que nas amostras do Estudo I e II a manifestação do diagnóstico do TEPT foi de $70 \%$, independente do instrumento utilizado para avaliação, apesar de estes apresentarem pequenas diferenças na estrutura e na forma de aplicação.

De um modo geral, as questões da SCID/TEPT parecem atender mais ao público adolescente e é aplicada com a vítima, enquanto que a K-SADS-PL/ TEPT aborda questôes mais voltadas ao público infantil e pode ser administrada tanto com a vítima quanto com um dos pais. Contudo, ambas se aplicam à pesquisa e à avaliação clínica de crianças e adolescentes vítimas de ASI e de outras situações traumáticas. Desta forma, os clínicos devem estar atentos às especificidades de cada instrumento na avaliação da escolha de um ou outro instrumento a ser utilizado.

Crianças reagem de diferentes formas aos eventos traumáticos. Por isso, profissionais da área da saúde devem estar familiarizados com os critérios de TEPT infantil e com seus aspectos específicos (Perrin et al., 2000). Sendo assim, há necessidade de ferramentas capazes de guiar uma boa avaliação, e estes instrumentos se mostraram ferramentas adequadas.

Novos estudos podem contribuir para uma maior compreensão da manifestação dos sintomas de TEPT na infância, abarcando aspectos pertinentes tanto às definições teóricas quanto à identificação de sintomas do transtorno. Falhas metodológicas na avaliação do TEPT infantil podem produzir equívocos quanto à prevalência deste transtorno, no diagnóstico e na intervenção posterior (Kaminer et al., 2005).

Algumas limitações metodológicas podem ser destacadas no presente estudo, dentre elas: o número pequeno de participantes, a escolha das amostras por conveniência e a característica de serem estudos realizados com amostras clínicas não possibilitam uma maior generalização dos resultados. Assim, novos estudos com um número maior de participantes, com amostra randomizada e acompanhamento longitudinal podem fornecer conhecimento específico da evolução e/ou remissão dos sintomas.

Finalmente, intervenções precoces podem contribuir para um maior ajustamento emocional no pós-trauma e impedir a cronificação do TEPT. Para tanto, instrumentos de avaliação do TEPT infantil que apresentem elevados níveis de sensibilidade e especificidade podem colaborar na elaboração de propostas de intervenções psicoterápicas em criança vítima de ASI. Ressalta-se, ainda, que o número de instrumentos de avaliação dos sintomas e do diagnóstico de TEPT infantil ainda é escasso. 


\section{REFERÊNCIAS}

Ackerman, P. T., Newton, J. E. O., McPherson, W. B., Jones, J. G. \& Dykman, R. A. (1998). Prevalence of post traumatic stress disorder and other psychiatric diagnoses in three groups of abused children (sexual, physical, and both). Child Abuse \& Neglect, 22, 759-774.

American Psychiatric Association. (2002). Diagnostic and Statistical Manual of Mental Disorders (4a ed. revised). Washington, DC: APA.

Brasil, H. H. A. (2003). Desenvolvimento da versão brasileira da K-SADS-PL (Schedule for Affective Disorders and Schizophrenia for School Aged Children Present and Lifetime Version) e estudo de suas propriedades psicométricas. (Tese de Doutorado não publicada). Curso de Pós-Graduação em Psiquiatria e Psicologia Médica da Universidade Federal de São Paulo-USP, São Paulo.

Breslau, N. (2002). Epidemiologic studies of trauma, posttraumatic stress disorder, and other psychiatric disorder. Canadian Journal of Psychiatry, 47, 923-929.

Briere, J. \& Elliott, D. M. (2003). Prevalence and psychological sequelae of self-reported childhood physical and sexual abuse in a general population sample of men and women. Child Abuse \& Neglect, 27, 1205-1222.

Câmara Filho, J. W. S. \& Sougey, E. B. (2001). Transtorno de estresse pós-traumático: Formulação diagnóstica e questōes sobre comorbidade. Revista Brasileira de Psiquiatria, 23, 221-228.

Cohen, J. A. (2003). Treating acute posttraumatic reactions in children and adolescents. Society of Biologial Psychiatry, 53, 827-833.

Collin-Vézina, D. \& Hébert, M. (2005). Comparing dissociation and PTSD in sexually abused school-aged girls. Journal of Nervous and Mental Disease, 193(1), 47-52.

Del Ben, C. M., Vilela, J. A., Crippa, J.A., Hallak, J. E., Labate, C. M. \& Zuardi, A. W. (2001). Confiabilidade da entrevista estruturada para o DSM-IV - versão clínica traduzida para o português. Revista Brasileira de Psiquiatria, 23(3), 156-159.

Duarte, J. C. \& Arboleda, M. R. C. (2004). Sintomatologia, avaliação e tratamento do abuso sexual infantil. In: Caballo, V. (Ed.). Manual de psicologia clínica infantil e do adolescente: transtornos gerais (pp. 293-321). São Paulo: Santos.

Dyregrov, A. \& Yule, W. (2006). A review of PTSD in children. Child and Adolescent Mental Health, 11, 176-184.

Elliott, A. N. \& Carnes, C. N. (2001). Reactions of nonoffending parents to the sexual abuse of their child: A review of the literature. Child Maltreatment, 6, 314-331.

Famularo, R., Fenton, T., Kinscherff, R. \& Augustyn, M. (1996). Psychiatric comorbidity in childhood post traumatic stress disorder. Child Abuse \& Neglect, 20, 953-961. 
Gomes, R., Deslandes, S. F., Veiga, M. M., Bhering, C. \& Santos, J. F. C. (2002). Por que as crianças são maltratadas? Explicações para a prática de maus-tratos infantis na literatura. Cadernos de Saúde Pública, 18, 707-714.

Habigzang, L. F. (2006). Avaliação e intervenção psicológica para meninas vítimas de abuso sexual intrafamiliar. (Dissertação de Mestrado não publicada. Curso de Pós-Graduação em Psicologia do Desenvolvimento) Universidade Federal do Rio Grande do Sul, Porto Alegre/RS.

Hawkins, S. S. \& Radcliffe, J. (2006). Current measures of PTSD for children and adolescents. Journal of Pediatric Psychology, 31, 420-430.

Kaminer, D., Seedat, S. \& Stein, D. J. (2005). Post-traumatic stress disorder in children. World Psychiatry, 4(2), 121-125.

Kaufman, J., Birmaher, B., Brent, D., Rao, U., Flynn, C., Moreci, P., Williamson, D. \& Ryan, N. (1997). Schedule for affective disorders and schizophrenia for school-age children-present and lifetime version (K-SADS-PL): initial reliability and validity data. Journal of American Academy of Child and Adolescent Psychiatry, 36, 980-988.

Kendall-Tackett, K. A., Williams, L. M. \& Finkellor, D. (1993). Impact of sexual abuse on children: a review and synthesis of recent empirical studies. Psychological Bulletin, 113(1), 164-180.

Kristensen, C. H. (1996). Abuso sexual em meninos. (Dissertação de Mestrado não publicada). Curso de Pós-Graduação em Psicologia do Desenvolvimento, Universidade Federal do Rio Grande do Sul. Porto Alegre, RS.

Nurcombe, B. (2000). Child sexual abuse I: Psychopathology. Australian and New Zealand Journal of Psychiatry, 34(1), 85-91.

Paolucci, E. O., Genuis, M. L. \& Violato, C. (2001). A meta-analysis of the published research on the effects of child sexual abuse. The Journal of Psychology, 135(1), 17-36.

Perrin, S., Smith, P. \& Yule, W. (2000). Practitioner review: The assessment and treatment of post-traumatic stress disorder in children and adolescents. Journal of Child Psychology and Psychiatric, 41, 277-289.

Pine, D. S. \& Cohen, J. A. (2002). Trauma in children and adolescents: Risk and treatment of psychiatric sequelae. Biological Psychiatry, 51, 519-531.

Pynoos, R. S. (1992). Transtorno de estresse pós-traumático em crianças e adolescentes. In: Garfinkel, B. D., Carlson, G. A. \& Weller, E. B. (Orgs.). Transtornos psiquiátricos na infância e adolescência (pp. 53-65). Porto Alegre: Artes Médicas.

Ruggiero, K. J., McLeer, S. V. \& Dixon, J. F. (2000). Sexual abuse characteristics associated with survivor psychopathology. Child Abuse \& Neglect, 24, 951- 964.

Runyon, M. K. \& Kenny, M. C. (2002). Relationship of atribucional style, depression and post trauma distress among children who suffered physical or sexual abuse. Child Maltreatment, 7(3), 254-264. 
Runyon, M. K., Faust, J. \& Orvaschel, H. (2002). Differential symptom pattern of posttraumatic stress disorder (PTSD) in maltreated children with and without concurrent depression. Child Abuse \& Neglect, 26(1), 39-53.

Scheeringa, M. S., Wright, M. J., Hunt, J. P. \& Zeanah, C. H. (2006). Factors affecting the diagnosis and prediction of PTSD symptomatology in children and adolescents. American Journal of Psychiatry, 163, 644-651.

Saywitz, K. J., Mannarino, A. P., Berliner, L. \& Cohen, J. A. (2000). Treatment for sexually abused children and adolescents. American Psychologist, 55, 1040-1049.

Tyler, K. A. (2002). Social and emotional outcomes of childhood sexual abuse: A review of recent research. Aggression and Violent Behavior, 7, 567-589.

\section{Anexo}

Tabela 1. Prevalência do Diagnóstico e Descrição das Médias de Sintomas por Critérios do TEPT em Meninas Vitimas de ASI

\begin{tabular}{|lcrr|}
\hline Critério & & $\begin{array}{c}\text { Estudo I } \\
\mathbf{( n = 4 0 )}\end{array}$ & \multicolumn{1}{c|}{$\begin{array}{c}\text { Estudo II } \\
(\mathbf{n = 1 5})\end{array}$} \\
\hline Diagnóstico de TEPT & $\%$ & 72,5 & 73,3 \\
Critério B - Revivência & & $3,03(1,23)$ & $2,73(0,88)$ \\
Critério C - Esquiva e entorpecimento & $M(S D)$ & $3,50(1,57)$ & $2,93(1,10)$ \\
Critério D - Excitabilidade aumentada & & $3,45(1,26)$ & $3,60(1,99)$ \\
\hline Total de sintomas & & $9,98(3,13)$ & $9,27(3,43)$ \\
\hline
\end{tabular}

Tabela 2. Médias de Sintomas por Critérios do TEPT em Meninas Vitimas de ASI com Diagnóstico Completo de TEPT e sem Diagnóstico

\begin{tabular}{|lcccc|}
\hline & \multicolumn{2}{c}{ Estudo I } & \multicolumn{2}{c|}{ Estudo II } \\
\cline { 2 - 5 } & Com TEPT & Sem TEPT & Com TEPT & Sem TEPT \\
& $(n=29)$ & $(n=11)$ & $(n=11)$ & $(n=4)$ \\
\cline { 2 - 5 } Critério & $M(S D)$ & $M(S D)$ & $M(S D)$ & $M(S D)$ \\
\hline B - Revivência & $3,38(1,18)$ & $2,09(0,83)$ & $2,91(0,83)$ & $2,25(0,96)$ \\
C - Esquiva e entorpecimento & $4,21(1,18)$ & $1,64(0,67)$ & $3,45(0,69)$ & $1,50(0,58)$ \\
D - Excitabilidade aumentada & $3,79(1,05)$ & $2,55(1,37)$ & $4,36(1,75)$ & $1,50(0,58)$ \\
\hline Total de sintomas & $11,38(2,34)$ & $6,27(1,49)$ & $10,73(2,61)$ & $5,25(1,71)$ \\
\hline
\end{tabular}




\section{Tabela 3. Descrição dos Itens por Critérios do TEPT dos Instrumentos dos Estudos I e II}

\begin{tabular}{|c|c|c|}
\hline Critério & Estudo I & Estudo II \\
\hline B - Revivência & $\begin{array}{l}\text { 1. 1. Você lembra muito do abuso } \\
\text { sexual, mesmo quando você } \\
\text { não quer lembrar ou pensar } \\
\text { sobre ele? Essas lembranças } \\
\text { vêm a tua cabeça muitas } \\
\text { vezes? } \\
\text { 2. 2. Você tem sonhos sobre o } \\
\text { abuso? } \\
\text { 3. 3. Você tem a sensação de que } \\
\text { o abuso está acontecendo de } \\
\text { novo mesmo quando ele não } \\
\text { está acontecendo na realidade? } \\
\text { 4. } 4 \text {. O que acontece quando } \\
\text { alguma coisa te faz lembrar } \\
\text { do abuso? Que coisas te fazem } \\
\text { lembrar o abuso? } \\
\text { 5. Você percebe alguma mudança } \\
\text { no teu corpo como, por } \\
\text { exemplo, ficar suado, coração } \\
\text { bater forte ou acelerado ou } \\
\text { dificuldade para respirar } \\
\text { quando alguma coisa lembra } \\
\text { o abuso? }\end{array}$ & $\begin{array}{l}\text { 1. Já teve época em que o abuso } \\
\text { ficava vindo na sua cabeça a toda } \\
\text { hora? Com que freqüência? } \\
\text { 2. Depois que o abuso aconteceu, } \\
\text { você já teve muitos pesadelos } \\
\text { ou sonhos ruins? } \\
\text { 3. Em brincadeira, você faz de } \\
\text { conta que o abuso estava } \\
\text { acontecendo? Às vezes você } \\
\text { brinca de ou os } \\
\text { seus bonecos?Você viu alguma } \\
\text { sombra ou vulto? } \\
\text { 4. Você já teve crises nervosas } \\
\text { em que chegou a ficar "fora } \\
\text { do ar", se comportando como } \\
\text { se estivesse vivendo aquele } \\
\text { momento ruim de novo? Você } \\
\text { já teve a impressão de que } \\
\text { estava acontecendo outra } \\
\text { vez? } \\
\text { 5. Você já esteve em algum lugar } \\
\text { que fez você lembrar do abuso? } \\
\text { Você já viu pessoas na rua que } \\
\text { fizeram você lembrar do abuso? } \\
6 \text {. Quando você está em } \\
\text { um lugar ou vê pessoas que } \\
\text { relembra o abuso, como você se } \\
\text { sente? Isso faz você passar mal? }\end{array}$ \\
\hline $\begin{array}{l}\mathrm{C}-\text { Esquiva e } \\
\text { entorpecimento }\end{array}$ & $\begin{array}{l}\text { 1. Você faz algum esforço para } \\
\text { não pensar ou falar sobre o } \\
\text { abuso? } \\
\text { 2. Você deixa de fazer alguma } \\
\text { atividade, ir a algum lugar } \\
\text { ou encontrar pessoas que te } \\
\text { fazem lembrar do abuso? } \\
\text { 3. Existe alguma coisa sobre o } \\
\text { abuso que você não consegue } \\
\text { lembrar }\end{array}$ & $\begin{array}{l}\text { 1. Que tipo de coisa você já fez } \\
\text { para não pensar no abuso? } \\
\text { 2. Você me disse que algumas } \\
\text { vezes___a faz lembrar do } \\
\text { que aconteceu. Você tenta } \\
\text { evitar__ ? } \\
\text { Você consegue lembrar de tudo o } \\
\text { que aconteceu? }\end{array}$ \\
\hline
\end{tabular}




\section{Tabela 4. Descrição dos Itens por Critérios do TEPT dos Instrumentos dos Estudos I e II (continuação)}

\begin{tabular}{|c|c|c|}
\hline Critério & Estudo I & Estudo II \\
\hline $\begin{array}{l}\mathrm{C}-\text { Esquiva e } \\
\text { entorpecimento }\end{array}$ & $\begin{array}{l}\text { 4. Você diminuiu seu } \\
\text { interesse ou participação } \\
\text { em atividades que antes } \\
\text { eram importantes para } \\
\text { você? Que atividades } \\
\text { foram essas? } \\
\text { 5. Você se sente sozinha } \\
\text { mesmo quando tem } \\
\text { gente ao seu redor? } \\
\text { 6. Você tem dificuldade } \\
\text { em sentir emoções ou } \\
\text { tem dificuldade de } \\
\text { ter sentimentos sobre } \\
\text { qualquer coisa ou } \\
\text { pessoas? } \\
\text { 7. Houve alguma mudança } \\
\text { nos seus planos para } \\
\text { o futuro depois que o } \\
\text { abuso aconteceu? Que } \\
\text { mudanças foram essas? }\end{array}$ & $\begin{array}{l}\text { 3. Que tipo de coisa você já fez para } \\
\text { não pensar no abuso? } \\
\text { 4. Você me disse que algumas vezes _- } \\
\text { a faz lembrar do que aconteceu. } \\
\text { Você tenta evitar_ ? } \\
\text { 5. Você consegue lembrar de tudo o } \\
\text { que aconteceu? } \\
\text { 6. Desde que o abuso aconteceu, você } \\
\text { passou a ficar sem vontade de fazer } \\
\text { nada (desinteressado, entediado)? } \\
\text { 7. Você é de confiar nas pessoas? Você } \\
\text { é do tipo que gosta de estar na } \\
\text { companhia de pessoas, de amigos, } \\
\text { ou você prefere ficar mais sozinho? } \\
\text { Foi depois que o abuso aconteceu } \\
\text { ou você já era assim? } \\
\text { 8. Tem coisas que faz você ficar alegre? } \\
\text { Depois que o abuso aconteceu, você } \\
\text { mudou a sua maneira de sentir as } \\
\text { emoçôes ou de reagir? } \\
\text { 7. O que você quer ser quando } \\
\text { crescer e for adulto? }\end{array}$ \\
\hline $\begin{array}{l}\mathrm{D}- \\
\text { Excitabilidade } \\
\text { aumentada }\end{array}$ & $\begin{array}{l}\text { 1. 1. Você tem dificuldade } \\
\text { para pegar no sono ou } \\
\text { dormir a noite toda? } \\
\text { 2. 2. Você tem estado mais } \\
\text { irritada ou tem sentido } \\
\text { mais raiva do que antes? } \\
\text { 3. 3. Você tem dificuldade } \\
\text { para se concentrar, } \\
\text { prestar atenção? } \\
\text { 4. 4. Você fica alerta mesmo } \\
\text { quando não tem motivo? } \\
\text { 5. 5. Você se assusta } \\
\text { facilmente com barulhos } \\
\text { inesperados? }\end{array}$ & $\begin{array}{l}\text { 1. Depois que o abuso aconteceu, } \\
\text { você já teve dificuldade para pegar } \\
\text { no sono ou para dormir a noite } \\
\text { inteira? } \\
\text { 2. Depois que o abuso aconteceu } \\
\text { você ficou mais irritada ou mal- } \\
\text { humorada? } \\
\text { 3. Você consegue ficar prestando } \\
\text { atenção no que você está fazendo? } \\
\text { 4. Depois que o abuso aconteceu você } \\
\text { ficou mais atento ao que está à sua } \\
\text { volta? } \\
\text { 5. } 5 \text {. Você se assusta com qualquer } \\
\text { barulhinho? }\end{array}$ \\
\hline
\end{tabular}

Recebido em 16 de fevereiro de 2009 Aceito para publicação em 24 de março de 2010 